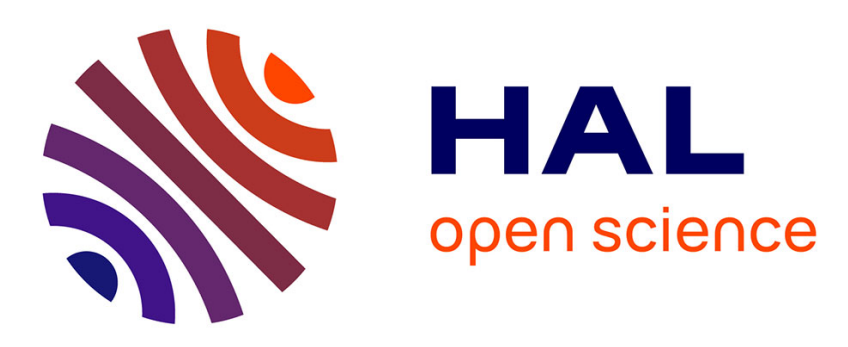

\title{
Monocyte CD169 expression as a biomarker in the early diagnosis of COVID-19
}

\author{
Anne-Sophie Bedin, Alain Makinson, Marie-Christine Picot, Frank
}

Mennechet, Fabrice Malergue, Amandine Pisoni, Esperance Nyiramigisha, Lise Montagnier, Karine Bollore, Ségolène Debiesse, et al.

\section{To cite this version:}

Anne-Sophie Bedin, Alain Makinson, Marie-Christine Picot, Frank Mennechet, Fabrice Malergue, et al.. Monocyte CD169 expression as a biomarker in the early diagnosis of COVID-19. Journal of Infectious Diseases, 2021, 223 (4), pp.562-567. 10.1093/infdis/jiaa724 . hal-03014885

\section{HAL Id: hal-03014885 \\ https://hal.science/hal-03014885}

Submitted on 28 Jul 2021

HAL is a multi-disciplinary open access archive for the deposit and dissemination of scientific research documents, whether they are published or not. The documents may come from teaching and research institutions in France or abroad, or from public or private research centers.
L'archive ouverte pluridisciplinaire HAL, est destinée au dépôt et à la diffusion de documents scientifiques de niveau recherche, publiés ou non, émanant des établissements d'enseignement et de recherche français ou étrangers, des laboratoires publics ou privés. 


\section{Monocyte CD169 Expression as a Biomarker in the Early Diagnosis of Coronavirus Disease 2019}

\begin{abstract}
Anne-Sophie Bedin,, ' Alain Makinson, ${ }^{2,3}$ Marie-Christine Picot, ${ }^{4,5}$ Frank Mennechet, ${ }^{1}$ Fabrice Malergue, ${ }^{6}$ Amandine Pisoni, ${ }^{1,7}$ Esperance Nyiramigisha, ${ }^{7}$ Lise Montagnier, Karine Bollore, ${ }^{1}$ Ségolène Debiesse, ${ }^{1}$ David Morquin, ${ }^{3}$ Nicolas Veyrenche, ${ }^{7}$ Constance Renault, ${ }^{1}$ Vincent Foulongne, ${ }^{1,7}$ Caroline Bret, ${ }^{8}$ Arnaud Bourdin, ${ }^{9,10}$ Vincent Le Moing, ${ }^{2,3}$ Philippe Van de Perre, ${ }^{1,7}$ and Edouard Tuaillon ${ }^{1,7}$

${ }^{1}$ Pathogenesis and Control of Chronic Infections, Montpellier University, INSERM, EFS, Montpellier, France, ${ }^{2}$ INSERM U1175/IRD UMI 233, IRD, Montpellier University, Montpellier, France, ${ }^{3}$ Department of Infectious Diseases, Montpellier University Hospital, Montpellier, France, ${ }^{4}$ INSERM, Centre d'Investigation Clinique 1411, Montpellier University, Montpellier France, ${ }^{5}$ Montpellier University Hospital, Montpellier, France, ${ }^{6}$ Department of Research and Development, Immunotech-Beckman Coulter, Marseille, France, 'Laboratory of Virology, Montpellier University Hospital, France, 'Laboratory of Hematology, Montpellier University Hospital, France, ${ }^{9}$ Department of Respiratory Diseases, Montpellier University Hospital, Montpellier, France, ${ }^{10}$ PhyMedExp, Montpellier University, CNRS, INSERM, Montpellier, France
\end{abstract}

We assessed the expression of CD169, a type I interferoninducible receptor, on monocytes (monocyte CD169 [mCD169]) in 53 adult patients admitted to the hospital during the coronavirus disease 2019 (COVID-19) outbreak for a suspicion of severe acute respiratory syndrome coronavirus 2 infection. Monocyte CD169 was strongly overexpressed in 30 of $32(93.7 \%)$ confirmed COVID-19 cases, compared with 3 of $21(14.3 \%)$ patients in whom the diagnosis of COVID-19 was finally ruled out. Monocyte CD169 was associated with the plasma interferon-alpha level and thrombocytopenia. Monocyte CD169 testing may be helpful for the rapid triage of suspected COVID-19 patients during an outbreak.

Keywords. CD169; COVID-19; monocytes; SARS-CoV-2; viral infections.

Early identification of coronavirus disease 2019 (COVID-19) and prompt diagnosis of severe acute respiratory syndrome coronavirus 2 (SARS-CoV-2) infection are critical for care and prognosis during outbreaks. It is also mandatory to avoid unnecessary and time-consuming interventions for ruling out COVID-19 in patients with serious respiratory conditions requiring appropriate care.

Type I interferons (IFNs) are important factors for homeostasis of the immune response and play a key role in antiviral immunity. A robust type-I IFN response is critical in the early phase of SARS-CoV-2 infection to limit SARS-CoV-2 replication and avoid severe complications [1]. CD169, also known as sialoadhesin or Siglec-1, is constitutively expressed at low levels on monocytes, but its expression rises dramatically when monocytes become stimulated by IFNa and all other type I IFNs. Monocyte CD169 (mCD169) overexpression is associated with acute viral infections $[2,3]$. Furthermore, a subset of CD169 lung-resident macrophages that have immune-regulatory functions and proliferate after influenza infection has recently been described [4].

In March 2020, Montpellier University Hospital reorganized its emergency service delivery in response to the COVID-19 outbreak to facilitate triage, diagnosis, and hospitalization of the patients presenting with suspected COVID-19. In this prospective observational study conducted during the COVID-19 outbreak, we evaluated mCD169 expression for the early identification of viral acute infection during SARS-CoV-2 epidemic in patients at hospital admission.

\section{METHODS}

This study was conducted by the University Hospital of Montpellier, during the peak of the COVID-19 epidemic between March 15 and April 5, 2020. It was approved by the accredited Institutional Review Board (IRB) No. 198711 (IRBMPT_2020_03_202000426). Each day, the first 15 consecutive adult patients presenting with a suspicion of COVID-19 and hospitalized in the medical units dedicated to the diagnosis and early care of COVID-19 were included in the study after written consent. Of the 53 eligible patients, 32 were confirmed to be SARS-CoV-2 positive using a nasopharyngeal swab, whereas 21 tested negative

. Among the confirmed cases, 18 patients had a second cytometry analysis before being discharged from the hospital. The median age of the patients was 64 years (interquartile range [IQR], 51-78.5 years) and $54.7 \%$ were male

\section{Ethical Approval Statement}

All patients were included in the COVIDOtheque cohort (ClinicalTrials.gov Identifier NCT04347850) and provided informed consent for the use of their data and clinical samples for the study. Institutional review board clearance for the scientific use of patient data has been granted by the Institution of Montpellier University Hospital and Ile de France III Ethical Committee (no. 2020-A00935-34).

\section{Flow Cytometry}

Ten microliters of ethylenediaminetetraacetic acid (EDTA) sample was simultaneously lysed with $500 \mu \mathrm{L}$ Versalyse lysing solution and stained with CD64-CD169/infections dried custom 
mixture, composed of anti-CD169-phycoerythrin (clone 7-239) and anti-CD64-Pacific Blue (PB) (clone 22) (Supplemental Figure 1). Results were expressed as CD169 monocyte/lymphocyte fluorescence ratio and CD64 neutrophils/lymphocytes fluorescence.

A second panel was used containing CD45-FITC, CD8ECD, CD3 PC5, CD4-RD1 (CYTO-STAT tetraCHROME), and $\mathrm{CD} 38$-PB. Samples were incubated at room temperature for 30 minutes in the dark. All products or custom products came from Beckman Coulter Inc. (Brea, CA). We acquired data on a 3-laser, 10-color Navios flow cytometer and analyzed data using Kaluza Software version 2.1 (both from Beckman Coulter Inc.).

\section{Positive Controls and Thresholds}

We assessed the expression of mCD169, nCD64, and CD38 bright on CD8 T cells in 30 healthy controls to establish a threshold based on the median +3 standard deviations. The thresholds were as follows: 3.51 for the $\mathrm{mCD} 169 /$ mean fluorescence intensity (MFI) ratio, 4.59 for the neutrophil CD64 (nCD64)/MFI ratio, and $9.06 \%$ for $\mathrm{CD} 38^{\text {bright }}$. The nCD64 threshold was controlled on 10 clinical samples collected from patients with microbiologically confirmed bacterial infections (median $=8.44$; IQR, 5.77-11.37) (data not shown).

\section{Severe Acute Respiratory Syndrome Coronavirus 2 Ribonucleic Acid Testing}

Severe acute respiratory syndrome coronavirus 2 ribonucleic acid (RNA) extraction from nasopharyngeal swabs (Sigma Virocult; Medical Wire Instrument, Corsham, UK) was done using the QIAamp Viral RNA Mini Kit on the QIAsymphony platform, following the manufacturer's instructions. Severe acute respiratory syndrome coronavirus 2 RNA was assessed using reverse-transcription polymerase chain reaction (RT-PCR) targeting RNA-dependent RNA polymerase as previously described.

\section{Interferon- $\alpha$ Plasma Concentration}

Plasma samples were stored at $-80^{\circ} \mathrm{C}$ until processing. Interferon- $\alpha$ was quantified using a multiplex microsphere assay (Invitrogen Human Inflammation 20-plex ProcartaPlex Panel, Marne-La-Vallée, France) on a Luminex apparatus (MAGPIX; Thermo Fisher Scientific, Waltham, MA) following the manufacturer's instructions.

\section{Serology}

Plasma samples were tested for immunoglobulin (Ig)G antibodies directed against SARS-CoV-2 nucleocapsid using a CE-marked enzyme-linked immunosorbent assay (ID Screen SARS-CoV-2-N; ID.Vet, Montpellier, France) as previously described [5]. Each result is displayed as a ratio: S/P (sample/ positive control) expressed in percentage (\%): $\mathrm{S} / \mathrm{P} \%=$ (optic density $[\mathrm{OD}]$ of sample-OD negative control)/OD positive control - OD negative control) $\times 100$. The $\mathrm{S} / \mathrm{P}>40 \%$ is positive and $<40 \%$ is negative.

\section{Statistical Analysis}

Data were analyzed and illustrated using Excel 2016 (Microsoft Corp., Redmond, WA) and Prism 7 (GraphPad Software Inc., La Jolla, CA) software. To determine statistical significance between the 2 groups (ie, CD169 COVID $^{+}$vs COVID $^{-}$), unpaired Student's 2-tailed $t$ test or non-parametric Mann-Whitney test was applied, according to the distribution of the data set. Correlations were analyzed using Pearson or Spearman's rank test according of the normality of the data set. A $P<.05$ was considered statistically significant.

\section{RESULTS}

There was a limit of 15 patients enrolled per day due to the limited capacity to include patients in the study. Among 162 patients admitted in the COVID-19 emergency unit from March 15 to April 5, 2020, 53 (32.7\%) were included and tested for mCD169 expression. Thirty-two of these patients tested positive for SARS-CoV-2 RNA, and 21 tested negative. Thirty patients with a confirmed diagnosis of COVID-19 had an mCD169 expression level above the positivity threshold (93.7\%) (Figure 1A). In contrast, only 3 of the 21 patients (14.3\%) with a negative SARSCoV-2 PCR result had an mCD169 overexpression. The level of mCD169 was inversely correlated with the cycle time detection (CT) value of the SARS-CoV-2 PCR, suggesting that the immunological marker was slightly associated with the level of virus replication $\left(R^{2}=0.24\right)$ (Figure 1B). At hospital admission, $\mathrm{mCD} 169$ was not associated with the duration, since the onset, of symptoms in COVID-19-confirmed patients (Supplemental Figure 2A). Likewise, the level of mCD169 expression had no prognostic value because no differences were noted in patients who had to be admitted in intensive care units, patients with mild forms of COVID-19, or patients who died (Figure 1B). Interferon- $\alpha$ concentration was significantly higher in COVID19-confirmed patients compared with COVID-19 negative patients $(P<.0001)$ (Figure 1D). Overall, the $\mathrm{mCD} 169$ level was correlated with the concentration of IFNa in plasma $(r=0.48)$ (Supplemental Figure 3F). The mCD169 expression level decreased during hospitalization in 15 of 18 confirmed COVID-19 patients, alongside IFNa concentration (Supplemental Figure 4). Of note, the platelet count increased between the first and second time points in all of the confirmed COVID-19 patients.

The mCD169 level was also negatively correlated with the platelet count $(\mathrm{r}=-0.45)$ (Supplemental Figure 3C), but no correlation was found with the $\mathrm{C}$-reactive protein (CRP) concentration, lymphocyte count, or neutrophil count (Supplemental Figure $3 \mathrm{~A}, \mathrm{~B}$, and E). To investigate whether mCD169 could complement serological testing, we retrospectively assessed anti-SARS-CoV-2 nucleocapsid IgG. Among the confirmed COVID-19 cases, 7 patients also tested positive for 

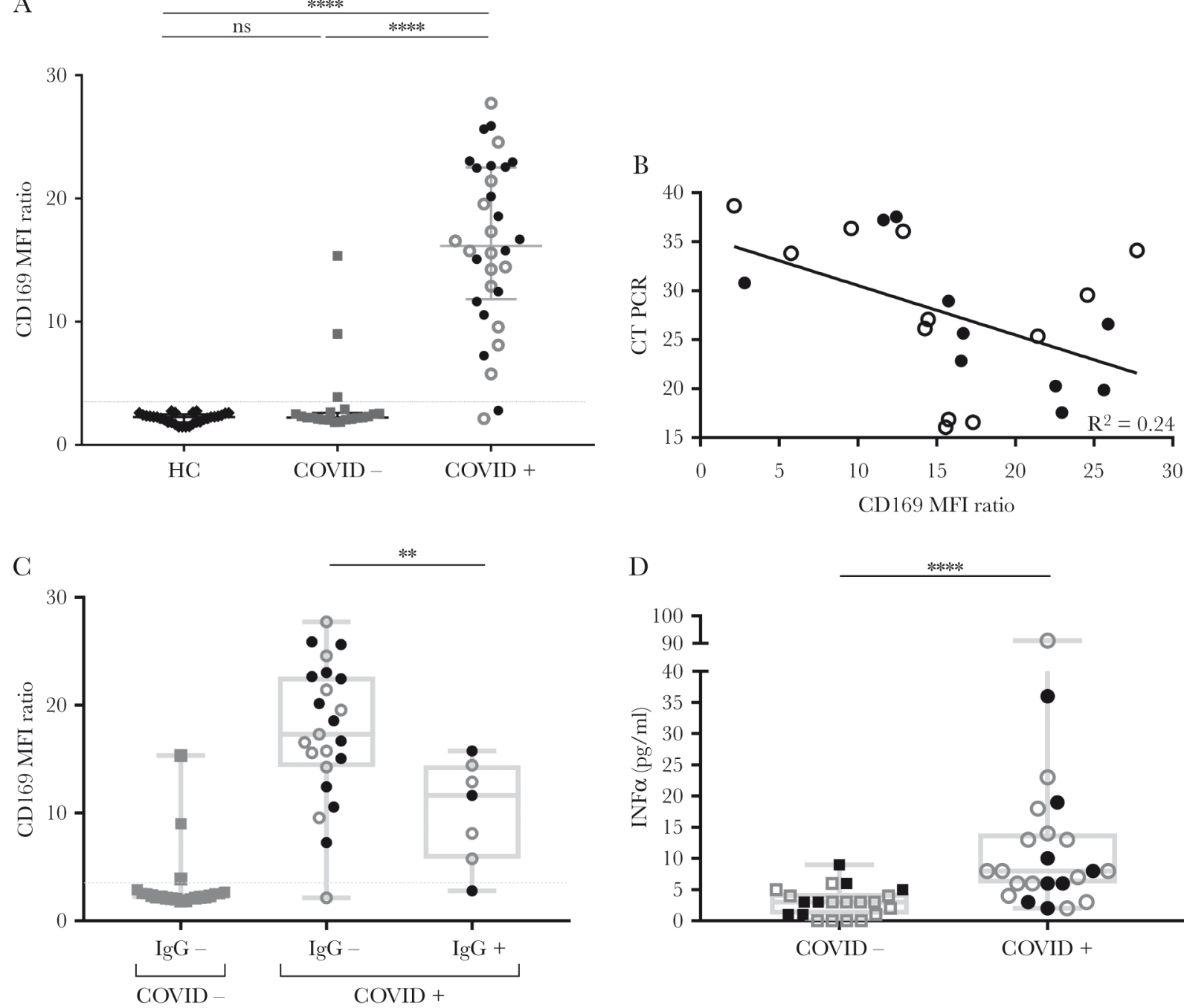

D

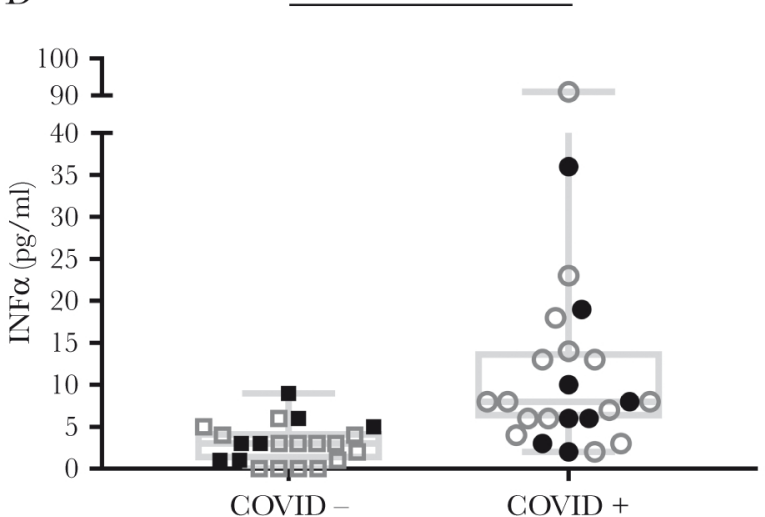

Figure 1. CD169 expression on monocytes in patients admitted in coronavirus disease 2019 (COVID-19) hospital units with a diagnosis of severe acute respiratory syndrome coronavirus 2 (SARS-CoV-2) infection confirmed or ruled out. (A) CD169 median of fluorescence intensity (MFI) ratio (CD169 on monocyte/CD169 on lymphocytes). Healthy controls are indicated by black diamonds; patients who tested negative for SARS-CoV-2 infection (COVID-) are indicated by open squares or black squares in patients with bacterial microbiologically confirmed infections; SARS-CoV-2 confirmed infection (COVID+) are open circles for mild and black circles for severe COVID-19. The threshold of CD169 MFI ratio is indicated by the dotted line: 3.51. ${ }^{* * *}, P<.0001$. (B) Correlation between CD169 MFI ratio and cycle time detection (CT) value of the SARS-CoV-2 reverse-transcriptase real-time polymerase chain reaction in COVID-19-confirmed patients. (C) Interferon- $\alpha$ (IFN $\alpha$ ) plasma concentration at hospital admission in patients with a diagnosis of SARS-CoV-2 infection confirmed or ruled out. ${ }^{*}, P<.0 .01$. (D) Expression of CD169 on monocyte according to serological status for anti-SARS-CoV-2 immunoglobulin $\mathrm{G}$ at hospital admission.

SARS-CoV-2 IgG at the time of hospital admission. These patients had a lower mCD169 level than patients who tested negative for anti-SARS-CoV-2 IgG $(P=.0084)$, suggesting that high mCD169 may be associated with active SARS-CoV-2 infection, before seroconversion (Figure 1C).

Neutrophil CD64 (nCD64) was also assessed as a marker for systemic bacterial infection [3]. High nCD64 expression was observed in 7 of 21 (33.3\%) SARS-CoV-2 PCR-negative patients (Supplemental Figure 2B). Bacterial infection was microbiologically confirmed for all but 1 of these patients (6 of 7). Four of 32 (12.5\%) confirmed COVID-19 cases also had nCD64 expression above the threshold; 2 of them had confirmation of a bacterial infection. A higher median expression of CD38 $8^{\text {bright }}$ on $\mathrm{CD}^{+} \mathrm{T}$ cells was also observed in the COVID-19-confirmed group compared with the SARS-CoV-2 uninfected group (Supplemental Figure 2C). However, this marker has a limited capacity to identified COVID-19 cases since CD $38^{\text {bright }}$ on $\mathrm{CD}^{+} \mathrm{T}$ cells was at a normal level in 11 COVID-19-confirmed cases $(34.3 \%)$. Nevertheless, it shows a strong correlation with CD169 ( $r=0.62)$ (Supplemental Figure 3D).

The test accuracy of mCD169 to predict COVID-19 infection during epidemic was studied by means of a receiver operating characteristic curve and compared with the CRP level (Figure 2). The sensitivity and specificity at the optimal operating point were $97 \%$ and $80 \%$, respectively, with an area under the receiver operating characteristic curve (AUC) of 0.95 . The CRP had a sensitivity and specificity of $94 \%$ and $33 \%$, respectively, with an AUC of 0.58 . 


\section{DISCUSSION}

The rapid identification of COVID-19 is a major challenge for emergency units, especially when a hospital has to cope with 200 to 300 suspected cases but a lower number of true SARSCoV-2 infections. Montpellier University Hospital has been strongly impacted by the COVID-19 crisis, although the region around Montpellier reported a relatively low proportion of COVID-19 cases during the first peak of the epidemic, which occurred in France between March and April 2020. Only a portion of patients suspected of COVID-19 had confirmation of the infection. For the remainder, the diagnosis of COVID19 needed to be rapidly ruled out after hospital admission. Monocyte CD169 may be a sensitive marker for the rapid triage of patients suspected of having COVID-19. Our observation on mCD169 overexpression in SARS-CoV-2-infected patients has been confirmed in other studies [6-8]. Type I IFNs induce the transcription of CD169 messenger RNA. Amplification of CD169 monocytes has been observed in other viral infections such as Zika, untreated human immunodeficiency virus, and influenza [9-11]. Hence, mCD169 may be a broad marker of viral infections. In this study, inclusions were carried out in March-April, whereas the influenza epidemic ended in week 11 in France [12]. The value of this marker in a context of a COVID and influenza virus double epidemic need to be explored and compared.

Overexpression appeared very early during COVID-19, but the dynamic of mCD169 remains to be explored. Patients tested

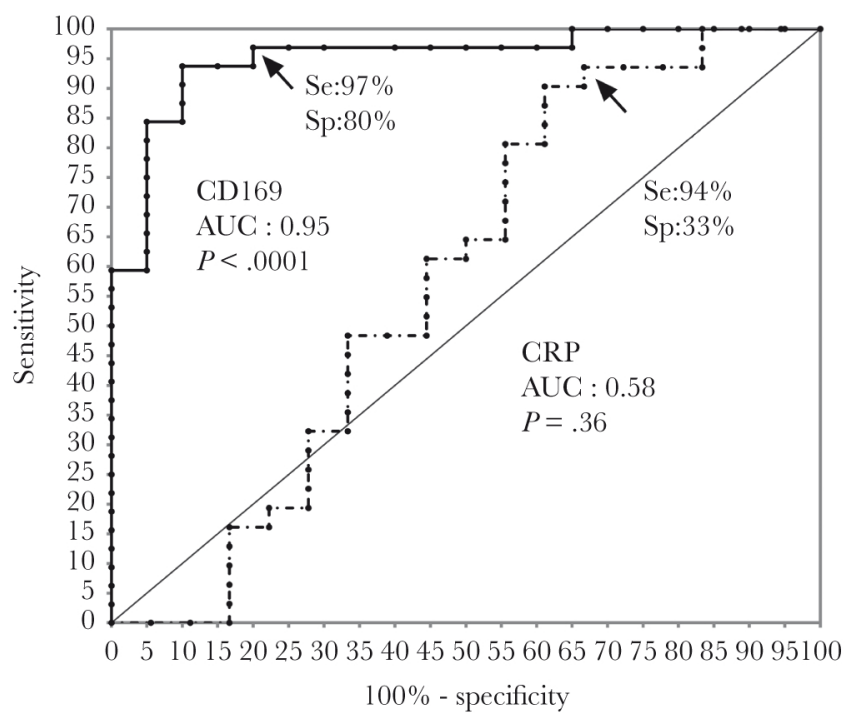

Figure 2. Receiver operating characteristic curve of CD169 median of fluorescence intensity (MFI) ratio and C-reactive protein (CRP) level for accuracy to predict coronavirus disease 2019 (COVID-19) infection. (1) Black line: COVID-19-positive and COVID-19-negative patients tested for CD169 MFI ratio. Area under the receiver operating characteristic curve $(A \cup C)=0.95 ; P<.0001 ; 95 \%$ confidence interval, 0.894-1.009. (2) Dotted line: COVID-19-positive and COVID-19-negative patients tested for CRP. The AUC of CRP $=0.58 ; P=.36$; $95 \%$ confidence interval, $0.39-0.76$. negative for anti-SARS-CoV-2 had lower mCD169 compared with anti-SARS-CoV-2-positive patient. Likewise, a trend for a lower level of mCD169 was observed in patients with high CT value and low plasma IFNa concentration. These results suggest that the expression of the marker decreased over the course of the disease.

Severe acute respiratory syndrome coronavirus 2 infection elicits type I IFN production, considered as key contributor to early innate response against COVID-19 [1]. Later in the disease course, impaired type I IFN production could be a characteristic of severe disease. As IFN-stimulated genes, transcription of CD169 may be lower in severe COVID compared with middle forms of the disease. A trend for a lower mCD169 expression has been reported in COVID-19-confirmed patients hospitalized in the intensive care unit. In our study, mCD169 at hospital admission was consistent across severity groups. The same observation was done using blood transcriptomes [14] and monocyte analysis [15] in patients with moderate versus severe COVID-19 outcomes.

\section{CONCLUSIONS}

In conclusion, $\mathrm{mCD} 169$ is strongly overexpressed on monocytes during the early phase of the SARS-CoV-2 infection, and it remains elevated in patients admitted to the hospital during the second week after the onset of symptoms. Alongside RT-PCR and serological testing, mCD169 may contribute to preserving the medical capacities of emergency departments by favoring the rapid orientation of patients with possible COVID-19. The value of leukocytes activation markers, including $\mathrm{mCD} 169$ and $\mathrm{nCD} 64$, in the diagnosis of acute infection needs to be evaluated during viral outbreaks in clinical studies. The development of fully automated tests for these markers may be crucial to prepare for future epidemics. 
1. Hadjadj J, Yatim N, Barnabei L, et al. Impaired type I interferon activity and inflammatory responses in severe COVID-19 patients. Science 2020; 369:718-24.

2. Kim WK, McGary CM, Holder GE, et al. Increased expression of CD169 on blood monocytes and its regulation by virus and CD8 T cells in macaque models of HIV infection and AIDS. AIDS Res Hum Retroviruses 2015; 31:696-706.

3. Bourgoin P, Lediagon G, Arnoux I, et al. Flow cytometry evaluation of infection-related biomarkers in febrile subjects in the emergency department. Future Microbiol 2020; 15:189-201.

4. Ural BB. et al. Identification of a nerve-associated, lungresident interstitial macrophage subset with distinct localization and immunoregulatory properties. Sci Immunol 2020; 5:eaax8756.

5. Tuaillon E, Bolloré K, Pisoni A, et al. Detection of SARS$\mathrm{CoV}-2$ antibodies using commercial assays and seroconversion patterns in hospitalized patients. J Infect 2020; 81:e39-45.

6. Chevrier S, Zurbuchen Y, Cervia C, et al. A distinct innate immune signature marks progression from mild to severe COVID-19. Available at: http://biorxiv.org/lookup/ doi/10.1101/2020.08.04.236315. Accessed 30 December 2020.

\section{Notes}

Acknowledgments. We thank S. Groc, S. Marie, and C. Niel for technical assistance. We also thank Grace Delobel for english language editing and review services.

Author contributions. A.-S. B. planned and supervised laboratory testing, analyzed the data, and wrote the manuscript. A. M., D. M., A. B., and V. L. M. managed patients and evaluated clinical data. A. P., L. M., E. N., K. B., S. D., V. F., and C. B. performed laboratory testing. F. M. wrote the manuscript. M.-C. P. evaluated clinical data and supervised statistical analysis. P. V. d. P. designed the study and wrote the manuscript. E. T. designed and conceptualized the study, analyzed the data, and wrote the manuscript.

Disclaimer. The funders had no role in the design of the study, the collection or analysis of data, or the decision to publish.

Financial support. This work was funded by the Montpellier University Hospital, Muse I-SITE Program Grant, University of Montpellier, and the Programme Hospitalier de Recherche Clinique Interrégional (PHRCI) Coro-TRI project.

Potential conflicts of interest. F. M. and P. B. are Beckman Coulter employees. All authors have submitted the ICMJE Form for Disclosure of Potential Conflicts of Interest. Conflicts that the editors consider relevant to the content of the manuscript have been disclosed.
7. Michel M, Malergue F, Belkacem IA. et al. An ultra-sensitive, ultra-fast whole blood monocyte CD169 assay for COVID19 screening. Available at: http://medrxiv.org/lookup/doi/1 0.1101/2020.10.22.20215749. Accessed 30 December 2020.

8. Roussel M, Ferrant J, Reizine F. et al. Mass cytometry and artificial intelligence define CD169 as a specific marker of SARSCoV2-induced acute respiratory distress syndrome. Available at: http://biorxiv.org/lookup/doi/10.1101/2020.09.22.307975. Accessed 30 December 2020.

9. Michlmayr D, Kim EY, Rahman AH, et al. Comprehensive immunoprofiling of pediatric Zika reveals key role for monocytes in the acute phase and no effect of prior dengue virus infection. Cell Rep 2020; 31:107569.

10. Sharma V, Bryant C, Montero M, et al; SEARCH007/ SEARCH011 Study Groups. Monocyte and CD4+ T-cell antiviral and innate responses associated with HIV-1 inflammation and cognitive impairment. AIDS 2020; 34:1289-301.

11. Bourgoin P, et al. CD64 and CD169 could help differentiate bacterial from viral infections in emergency department. Available at: http://medrxiv.org/lookup/doi/10.1101/2020. 10.28.20221259. Accessed 30 December 2020.

12. Santé publique France, Bulletin epidemiologique grippe. Bilan de la surveillance, saison 2019-2020. Available at: https:// www.santepubliquefrance.fr/maladies-et-traumatismes/ 
maladies-et-infections-respiratoires/grippe/documents/ bulletin-national/bulletin-epidemiologique-grippe-bilande-la-surveillance-saison-2019-2020. Accessed 30 December 2020.

13. Carissimo G, Xu W, Kwok I, et al. Whole blood immunophenotyping uncovers immature neutrophilto-VD2 T-cell ratio as an early marker for severe COVID19. Nat Commun 2020; 11:5243.
14. Aschenbrenner AC. Mouktaroudi M, Kraemer B, et al. Disease severity-specific neutrophil signatures in blood transcriptomes stratify COVID-19 patients. Available at: http://medrxiv.org/lookup/doi/10.1101/2020.07.07.201483 95. Accessed 30 December 2020.

15. Silvin A, Chapuis N, Dunsmore G, et al. Elevated calprotectin and abnormal myeloid cell subsets discriminate severe from mild COVID-19. Cell 2020; 182:1401-18. 